\title{
Analytical Solutions to Evaluate Bending Failure of Column Supported Embankments
}

\author{
N. N. S. Yapage, D. S. Liyanapathirana, H. G. Poulos, R. B. Kelly, and C. J. Leo
}

\begin{abstract}
Design procedures for Geosynthetic Reinforced Column Supported (GRCS) embankments generally consider the sliding failure for external stability and the shear failure mode for internal stability. However, other failure modes such as collapse failure, slip circle failure, punching shear failure around column heads (overall or local), and bending failure of DCM columns are also significant for GRCS embankments. Although some design procedures have considered some of these failure modes and proposed design criteria to check against these failure modes, they are not currently used in practice due to uncertainties related to some assumptions used in developing these methods, unreliable results given by them and difficulty in finding some parameters. Still there are uncertainties in identifying the critical failure modes for these embankments at the ultimate limit state (ULS) conditions. In this paper, two-dimensional finite element modeling incorporating strain softening behavior of cement admixed soils is used to identify the failure modes of GRCS embankments at the ULS. Bending failure of columns with subsequent shear failure is found to be critical for internal stability of GRCS embankments. Consequently, analytical equations are derived to calculate the factor of safety against bending failure for improved ground considering driving and resisting moments.
\end{abstract}

Index Terms-Deep cement mixed columns, embankments, finite element method, failure modes, strain softening.

\section{INTRODUCTION}

Deep cement mixed (DCM) column-supported embankments, with or without geosynthetic reinforcement, are widely used in marginal and soft ground with poor soil conditions to elevate the platform for highways and runways. Numerous studies based on physical modeling (full scale field tests or centrifuge tests) and numerical modeling have been used extensively to understand the behavior of these complex structures.

Investigation of GRCS embankment behavior using numerical modeling, based on the finite element or finite difference method, is economical and less time consuming compared to experimental methods employing centrifuge or physical modeling. Therefore two and three-dimensional finite element modeling techniques are widely used to enhance the understanding of the behavior of geosynthetic

Manuscript received February 10, 2013; revised May 23, 2013. This work was supported in part by the Australian research council and Coffey Geotechnics Pty Ltd under the Linkage project LP0990581. Analytical Solutions to Evaluate Bending Failure of Column Supported Embankments.

N. N. S. Yapage, D. S. Liyanapathirana and C. J. Leo are with School of Computing, Engineering and Mathematics, University of Western Sydney, Locked Bag 1797, Penrith, NSW 2751, Australia (e-mail: n.yapage@ uws.edu.au, s.liyanapathirana@uws.edu.au, and c.leo@uws.edu.au).

H. G. Poulos and R. B. Kelly are with Coffey Geotechnics Pty Ltd, Level 19, Tower B, Citadel Tower 799, Pacific Highway, Chatswood, NSW 2067, Australia (e-mail: Harry_Poulos@coffey.com, and Richar_Kelly@coffey.com). reinforced column supported (GRCS) embankments [1]-[7] However modeling of DCM columns with an elastic perfectly plastic Mohr-Coulomb model is insufficient to investigate the overall behavior of embankments beyond the yielding of the cement admixed columns.

In current design practice it is assumed that the performance of GRCS embankments is similar to pile-supported embankments and the constitutive behavior of DCM columns are simulated assuming elastic-perfectly plastic behavior [1], [2], [5]-[7]. There are significant differences between the constitutive behaviors of cement admixed soils and concrete commonly used for pile-supported embankments. The main difference is the strain softening behavior of cement admixed soils due to progressive degradation of the cement-soil structure beyond yielding. Therefore, if yielding occurs in the analysis, numerical models with linear elastic perfectly plastic column behavior may lead to underestimation of the total and differential settlements, unrealistic failure patterns and overestimation of bearing capacities [8]. It is essential to consider progressive failure when these embankments are being designed for ultimate limit state conditions. Reference [8] discussed the importance of the consideration of progressive failure of cement admixed soil in the limit state design procedures for GRCS embankments. Also they have demonstrated the importance of its incorporation to the numerical models to identify ultimate limit state failure modes of GRCS embankments as compared to pile supported embankments.

Few design procedures are available in the literature for GRCS embankments (e.g. [9] and [10]). In them, internal and external stability analyses are considered, but these design procedures for GRCS embankments only consider the sliding failure for external stability and the shear failure mode for internal stability. Using centrifuge model tests, references [11]-[14] found that various failure modes such as collapse failure (a somewhat domino type failure), slip circle failure, punching shear failure around column heads (overall or local), and bending failure of DCM columns are also significant for ground improved with a group of DCM columns under embankment loading. References [15]-[17] have considered some of these failure modes and proposed new design methods. However analytical equation proposed by [15] and [16] are not currently used in practice due to uncertainties related to some assumptions used in developing these methods, unreliable results given by them and difficulty in assessing some parameters. Equations proposed by [17] can be refined to consider the critical failure plane, traffic load and the geosynthetic.

In this paper, it is shown that the bending failure and subsequent slip surface shear failure are critical for internal 
stability of GRCS embankments using 2D finite element analysis incorporating strain softening behavior of DCM columns. Consequently analytical solutions are derived and discussed to calculate the factor of safety for this failure mode.

\section{IDENTIFICATION OF CRITICAL FAILURE MODE FOR GRCS EMBANKMENTS USING 2D NUMERICAL MODELING}

In a non-linear finite element analysis, the initiation, development and distribution of a potential plastic or shear strain indicate the propensity of failure modes. It is found that the bending failure and subsequent slip surface shear failure are critical for internal stability of GRCS embankments using $2 \mathrm{D}$ finite element analysis. The plastic hinge formation, shear strain distribution, and the slip surfaces development within the finite element model are shown in Fig. 1 and Fig. 2. Modeling procedure, material models and parameters are given in [8]. When the development of shear strain with gradual loading is investigated, higher shear strains initially develop closer to the top of the columns at the center of the embankment and then they progressively develop towards the bottom of the columns closer to the embankment toe, as shown in Fig. 1.

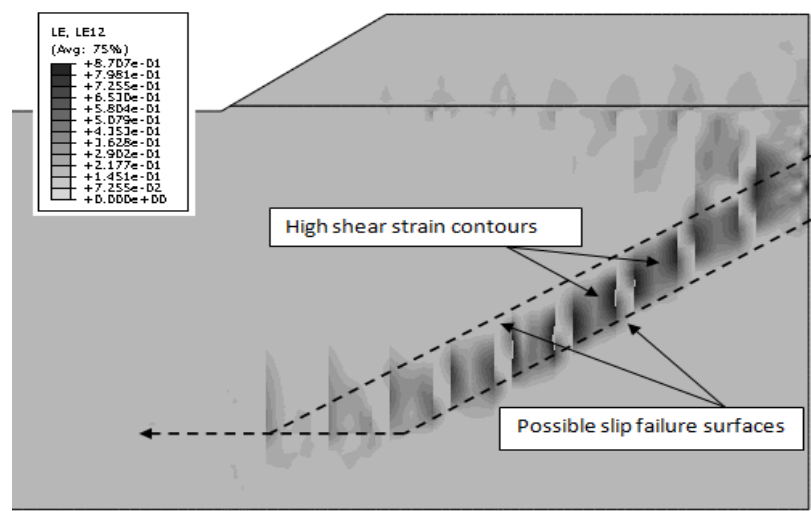

Fig. 1. Equivalent shear strain distribution of the embankment in numerical analysis when symmetric nature is considered [8].

During this process, DCM columns in the active zone fail one by one from the bending failure mode. When the maximum bending moments within the columns exceed the moment carrying capacity of columns, plastic hinges will develop at these locations, as illustrated in Fig. 2. The soft soil in between these columns experiences considerable shear distortions due to abrupt deformation of these damaged columns. Finally this phenomenon produces critical slip surfaces as shown in Fig. 1 and Fig. 2. The combination of resulting failure slip surfaces is not circular but a slip band with a certain thickness as shown in Fig. 1. During the formation of this critical slip surfaces, columns in the active shear zone can fail by shearing due to the considerable horizontal displacement.

No failure occurs in the bottom stiff layer but the failure zone passes through the base of the soft clay layer. This failure mechanism is similar to the mechanism described by [16] for the columns in the active zone but considered only an average slip surface as shown in Fig. 3. Below the centre of the embankment, closer to the top of the soft clay layer, two failure envelopes merge as shown in Fig. 3 and it is confirmed by the 2D numerical model [18].

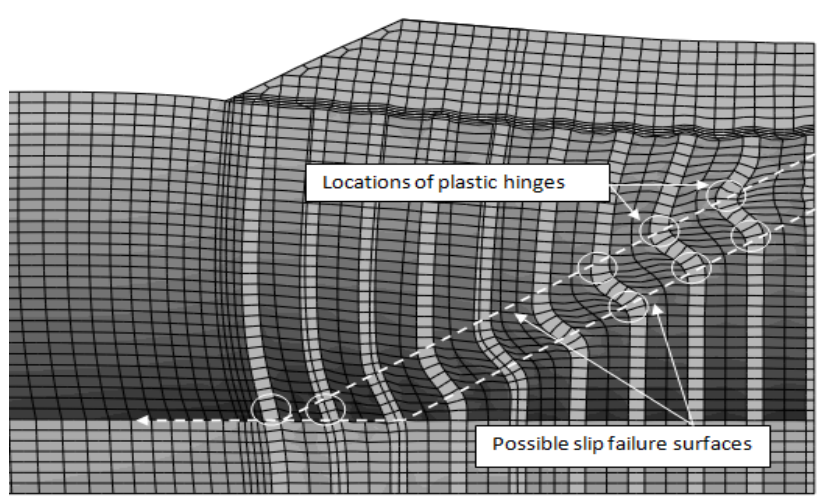

Fig. 2. Deformed shape of the finite element model.

Although this failure mode is a combination of bending and shear failure modes, in this paper, for simplicity, the stability calculations are proposed separately to evaluate the bending and shear failure modes. Due to the page limitations for this paper, only the analytical equations derived for the stability calculation of bending failure are presented.

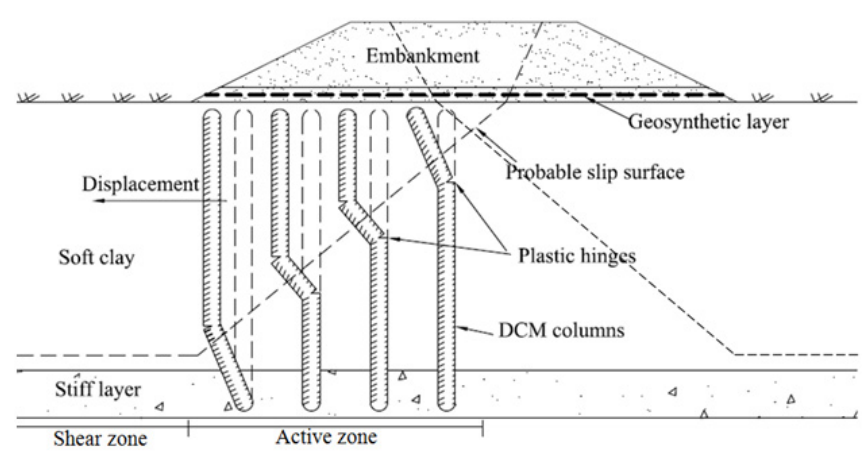

Fig. 3. Failure mode of an embankment for internal stability (after [16]).

\section{Design OF GRCS EMBANKMENTS AgAinst BENDING FAILURE}

Reference [17] proposed a simple stability calculation to assess embankments over improved ground against ultimate bending failure. However, he has not considered the influence of traffic load over the crest and the tension developed within the geosynthetic layer. He assumed that the envelope of failure plane of columns is horizontal. However, the failure plane is an inclined one as shown in Fig. 1, Fig. 2 and Fig. 3. Therefore, a new stability equation should be developed for the bending failure mode, incorporating the inclined failure plane. In the analytical solution, the active earth pressure due to the embankment load, Pae, and soft clay, Pac, as well as the traffic load, Pat, should be considered to calculate the driving moment. The resisting moment consists of the contributions from passive earth pressure of the soft clay, Ppc, embankment and traffic load over the columns, Pel, self-weight of columns, Psw, tension in the geosynthetic, Tgs, the skin friction mobilized along the surface of columns, Fsf, and the shear strength of clay between columns, Fsc. For the calculation of driving and resisting moments due to active and passive earth pressures of the clay ground respectively, all soil wedges should be considered individually as shown in Fig. 4. The resultant of driving and resisting moments due to 
loads applied on columns should not exceed the bending strength of DCM columns.

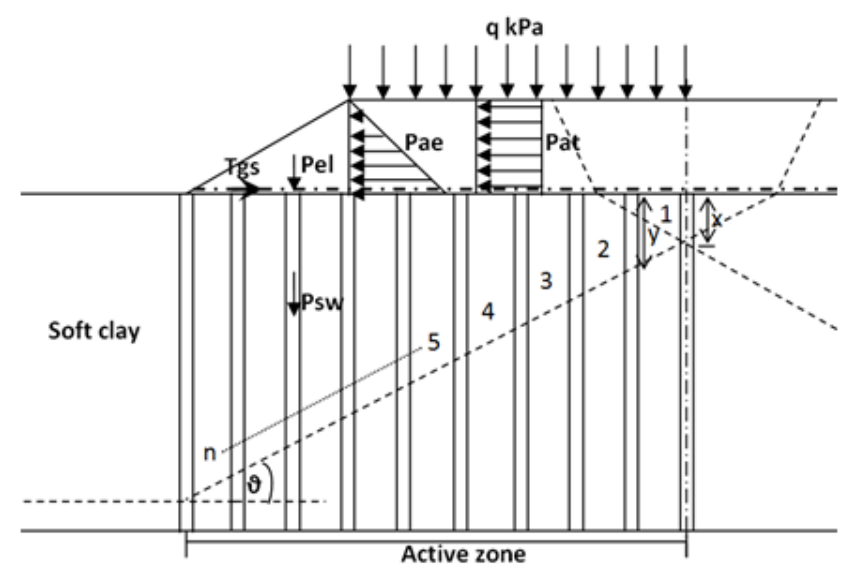

Fig. 4. Load distribution over columns for embankment in consideration for the bending failure analysis.

\section{Calculation of FOS for Bending Failure}

A systematic stability calculation is presented in this section. It is assumed that all DCM columns fail simultaneously in bending and the active zone ends vertically below the toe of the embankment. In addition, the improved area above the failure plane deforms or moves over the failure plane, as shown in Fig. 4. The top slip surface is found to initiate first from the FE analysis (Fig.1 and Fig. 2) and therefore derivations are based on that. The inclined failure plane is initiated at the center line at depth $\mathrm{x}$, for a wide embankment, and it ends vertically below the toe of the embankment similar to the top slip surface. However $x$ becomes zero for narrow embankments. The moment equilibrium at the failure plane can be calculated as described in the following sections. Both driving moments and resisting moments are calculated per unit width of the embankment in the longitudinal direction.

\section{A. Driving Moment Calculations}

Driving moments only occur due to the active earth pressure applied by the ground, active earth pressure from the embankment and traffic load. For the calculation of FoS for bending failure, only the active zone is considered.

1) Driving moment from the active earth pressure of the improved ground, $M_{a c}$

Driving moment per unit width in the longitudinal direction by active earth pressure is calculated using Coulomb's method of active pressure computation based on the wedge theory for retaining walls. Therefore these calculations can be classed as limit equilibrium methods. The assumed failure surface is shown in Fig. 4, which is similar to the failure surface given by the numerical analysis. The shear stresses developed at the column-soil interface are assumed to be fully mobilized but not strained softened. To calculate the active earth pressure, soil wedge slices between columns are considered. It is assumed that only one side of the soil wedge touches the DCM columns. This assumption is reasonable because when the soil is in a state of plastic equilibrium, the wedge of soil between two adjacent columns is about to move. Then the DCM column at the opposite side of the direction of motion will break away from the soil. At the same time, the soil wedge is about to break away from the main clay ground. Calculations are not shown step by step in what follows, but only the final solution is given due to the page limit of this paper.
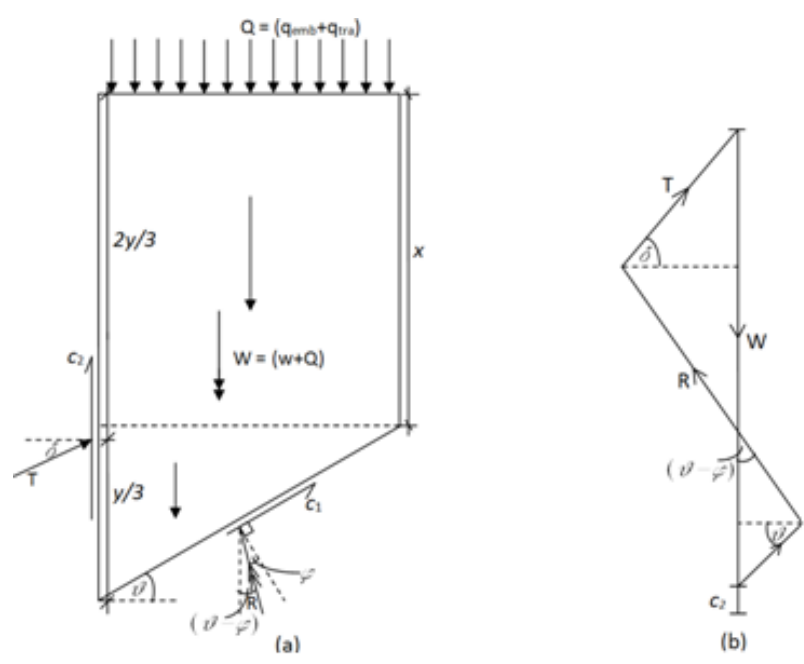

Fig. 5. (a). General soil wedge between two columns for the calculation of active earth pressure (b). Force polygon

All forces acting on the soil wedge are shown in Fig. 5(a) and the force polygon for the equilibrium state is illustrated in Fig. 5 (b). From that, force due to active earth pressure or the thrust applied on the column from the soil wedge, T, can be found. Then the driving moment $M_{a c}$ around the failure plane can be found. Finally Equation 1 is derived using summation of a finite series for the active earth pressures from all soil wedges.

$$
\begin{aligned}
& M_{a c}=\sum_{i=1}^{(n-1)} M_{i}=P\left[\begin{array}{l}
\left.A\left[\begin{array}{l}
(n-1) 2 x^{2}+2 x s(n-1)^{2} \tan \theta+ \\
s^{2} \frac{n(n-1)(4 n-5)}{6} \tan ^{2} \theta
\end{array}\right]+\right] \\
{\left[\begin{array}{l}
\left.(n-1) x+\frac{n(n-1) s}{2} \tan \theta\right] \\
2
\end{array}\right]} \\
C\left[\begin{array}{l}
(n-1) x^{2}+n(n-1) x s \tan \theta+ \\
\frac{n(n-1)(2 n-1)}{6} s^{2} \tan ^{2} \theta
\end{array}\right]
\end{array}\right] \\
& A=\frac{s \gamma}{2 \cos (\theta-\phi)}, \quad, \quad B=\frac{q_{t r a}+q_{e m b}}{\cos (\theta-\phi)}-\frac{2 \cos \phi \times s \times c_{s}}{\cos \theta \times \sin ^{2}(\theta-\phi)} \\
& C=\frac{c_{c o l}}{\cos (\theta-\phi)}, P=\frac{y \cos \delta \times \sin 2(\theta-\phi)}{6 \cos (\delta-\theta+\phi)}
\end{aligned}
$$

2) Driving moment from the active earth pressure of the embankment, $M_{a e}$

$$
\begin{aligned}
& M_{a e}=\gamma_{e} \frac{H_{e}{ }^{2}}{6} \tan ^{2}\left(\pi / 4-\phi_{e} / 2\right) \times \\
& {[1+2 n x+n(n-1) s \tan \theta]}
\end{aligned}
$$

3) Driving moment from the traffic load of the embankment, $M_{a t}$ 


$$
\begin{aligned}
& M_{a t}=\left(n x+\frac{n(n-1) s}{2} \tan \theta+\frac{H_{e}}{2}\right) \times \\
& q H_{e} \tan ^{2}\left(\pi / 4-\phi^{e} / 2\right)
\end{aligned}
$$

\section{B. Resisting Moment Calculations}

In this section, resisting moment calculations for each force component are presented. Resisting moment from passive earth pressure is calculated similar to the active earth pressure calculations. Forces acting on an arbitrary soil wedge considered for the derivation are shown in Fig. 6.

1) Due to tension in geosynthetic, $M_{T g s}$

For a single column row;

$$
M_{T g s}=T_{g s} \times z \times \frac{1}{s}
$$

For the whole improved ground;

$$
\sum_{i=1}^{n} M_{i}=T_{g s} \times \frac{1}{s}\left[n x+\frac{n(n-1) s}{2} \tan \theta\right]
$$

2) Due to passive earth pressure of the ground, $M_{p e}$

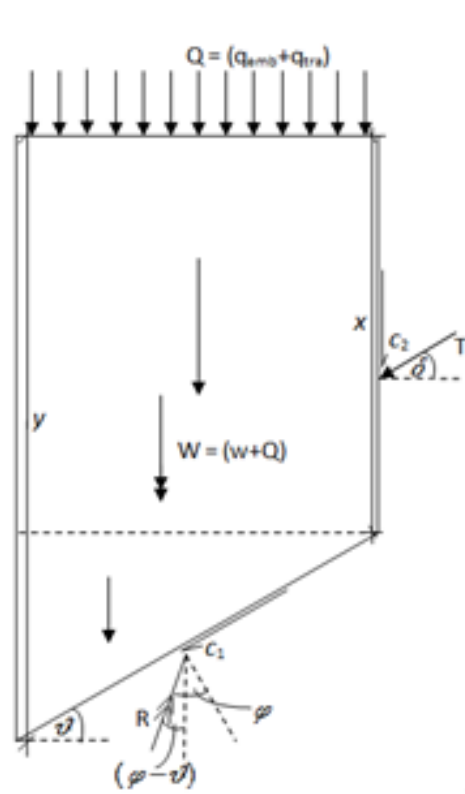

(a)

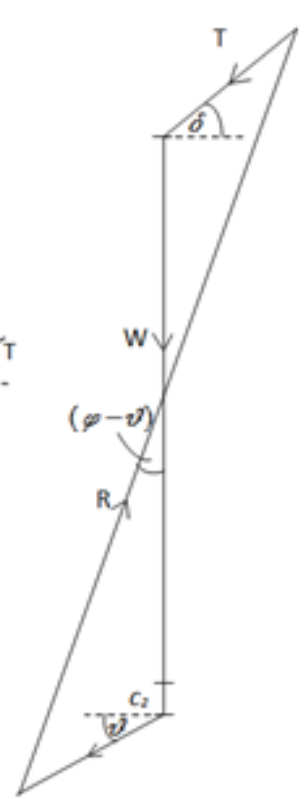

(b)
Fig. 6. (a) General soil wedge between two columns for the calculation of passive earth pressure (b) Force polygon

$M_{p e}=\sum_{i=1}^{(n-1)} M_{i}=A\left[\begin{array}{l}B\left[\frac{(n-1) x+(n-2)(n-1) s \tan \theta}{3}\right] \\ \frac{C}{6}\left[\begin{array}{l}2(n-1) x^{2}+(n-1)^{2} x s+ \\ \frac{(n-1)(n-2) s x \tan \theta+}{6}(n-2)(4 n-3) \\ \frac{(n-1)}{6} \tan ^{2} \theta\end{array}\right]\end{array}\right]$

$$
\begin{aligned}
& A=\frac{\cos \delta}{\cos (\delta+\phi-\theta)}, \quad B=\sin (\phi-\theta)\left[c_{2}+c_{1} \times \sin \theta+Q\right]- \\
& c_{1} \cos \theta \cos (\phi-\theta) \\
& Q=q_{t r a}+q_{e m b}, C=s \gamma \sin (\phi-\theta)
\end{aligned}
$$

3) Due to bending strength of columns, $M_{p b}$

$$
M_{p b}=\sum_{i=1}^{n} M_{p b, i}=\frac{\pi D^{3}}{32} \times \alpha \times q_{u} \times N \times \frac{1}{s}
$$

4) Due to self-weight of columns, $M_{\text {pself }}$

$$
\begin{aligned}
& M_{p s e l f}=\sum_{i=1}^{n} M_{p s e l f, i}=\frac{\pi D^{3}}{8} \times \gamma_{c o l} \times N \times \\
& \frac{1}{s}\left[n x+\frac{(n-1) n s}{2} \tan \theta\right]
\end{aligned}
$$

5) Due to embankment load, $M_{\text {pemb }}$

$$
\begin{aligned}
& M_{p e m b}=\sum_{i=1}^{n} M_{p e m b, i}=\frac{\pi D^{3}}{8} \times H_{e} \times \gamma_{e} \\
& \times \mu_{c o l} \times N \times \frac{1}{s}
\end{aligned}
$$

6) Due to adhesion mobilized on the side surface of DCM columns, $M_{r c}$

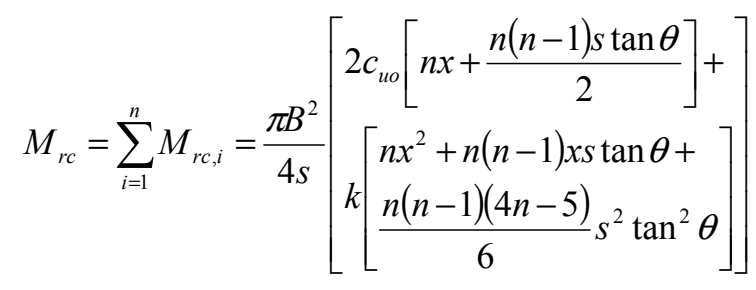

7) Due to shear strength of clay between columns, $M_{s c}$

$$
M_{s c}=s \times\left(1-a_{s}\right) \times \frac{2 \cdot c_{u 0}+k \times z}{2} \times(N-1) \times z
$$

Finally, FoS can be calculated using Equation 13, which is derived by substituting all driving and resisting moments to Equation (12).

$$
\begin{aligned}
& F O S=\frac{\text { Resisting moments }}{\text { Driving moments }} \\
& F o S=\frac{\left[\begin{array}{l}
M_{T g s}+M_{p e}+M_{p b}+M_{p s e l f}+ \\
M_{p e m b}+M_{r c}+M_{s c}
\end{array}\right]}{\left[M_{a e}+M_{a c}+M_{a t}\right]}
\end{aligned}
$$

\section{CONCLUSION}

Finite element analyses show that bending failure is the most critical failure mode for internal stability of GRCS embankments. Once plastic hinges are formed due to the moment capacity of the columns being exceeded, the embankment fails due to propagation of a slip surface, which is mainly governed by the tensile strength of the columns and to some extent, the shear strength of the unstabilized clay. Analytical equations for the stability calculation against 
bending failure considering all possible driving and resisting moments are derived. It is possible to have an infinite number of planes. Hence, the main problem is to determine the angle of critical failure plane and the point where the critical failure plane initiates. They can be determined by carrying out several trial computations, considering different failure planes to obtain the maximum thrust or minimum FoS. It is important to determine how the gradient and position of this failure line vary with geometry and the material properties of the embankment. To achieve this, a detailed parametric study will be carried out in future research.

\section{NOTATIONS}

$\begin{array}{ll}w & : \text { Force due to mass of the soil wedge } \\ q_{\text {emb }} & : \text { Embankment load over the soil wedge } \\ q_{t r a} & : \text { Traffic load over the soil wedge } \\ W & : w+q_{\text {emb }}+q_{\text {tra }} \\ n & : \text { Number of soil wedges } \\ \theta & : \text { Angle of the failure plane to horizontal level } \\ c_{1} & : \text { Cohesive force between wedge and rest of the } \\ c_{2} & : \text { clay ground } \\ R & : \text { Reaction between clay ground and wedge acting } \\ & \text { at } \phi \text { to the normal to slip plane }\end{array}$

$T \quad$ : Trust reaction from columns acting at $\delta$ to the normal to column surface

$\phi \quad$ : Angle of shearing resistance of soft soil

$\phi_{e} \quad$ : Angle of shearing resistance of fill material

$\gamma_{e} \quad$ : Unit weight of the fill material

$\gamma \quad:$ Unit weight of the soft soil

$\delta \quad:$ Angle of friction between soil and DCM columns

$a_{s}:$ Area replacement ratio

$D \quad$ : Width of the improved ground

$B \quad$ : Diameter of columns

$S \quad$ : Column spacing

$N \quad$ : Number of DCM columns rows

$\alpha \quad$ : Bending strength ratio to UCS

$\sigma_{b} \quad$ : Bending strength

$\mu_{s} \quad$ : Stress concentration coefficient

$q_{u} \quad$ :Unconfined compressive strength of DCM columns

$c_{u 0} \quad:$ Undrained shear strength at the ground level

\section{ACKNOWLEDGMENT}

The authors would like to acknowledge the financial support for this research provided by the Australian research council and Coffey Geotechnics Pty Ltd under the Linkage project LP0990581

\section{REFERENCES}

[1] J. Huang, J. Han, and S. Oztoprak, "Coupled mechanical and hydraulic modeling of geosynthetic-reinforced column-supported embankments," Journal of Geotechnical and Geoenvironmental Engineering, vol. 135, no. 8, pp. 1011-1021, 2009.

[2] J. Huang and J. Han, "3D coupled mechanical and hydraulic modeling of a geosynthetic-reinforced deep mixed column-supported embankment," Geotextiles and Geomembranes, vol. 27, no. 4, pp. 272-280, 2009.

[3] R. S. Madhyannapu, A. J. Puppala, S. Hossain, J. Han, and A. Porbaha, "Analysis of geotextile reinforced embankment over deep mixed soil columns: Using numerical and analytical tools," GeoCongress 2006: Modelling and Characterization of Deep Soil Cement, Atlanta, GA, 248, 2006

[4] M. P. Navin and G. M. Filz, "Statistical analysis of strength data from ground improved with DMM columns," Deep Mixing '05: International Conference on Deep Mixing Best Practice and Recent Advances, pp. 145-154, 2005.

[5] M. P. Navin and G. M. Fliz, "Numerical stability analysis of embankments supported on deep mixed columns," in Proc. ASCE Conf. Ground Modification and Seismic Mitigation (GSP 152), 2006, pp. 1-8.

[6] M. E. Stewart, M. P. Navin, and G. M. Fliz, "Analysis of a column-supported test embankment at the I-95/route 1 interchange," Geotechnical Engineering for Transportation Projects, Geo-Trans 2004 (GSP 126), 2004, pp. 1337-1346.

[7] G. Fliz, "Load transfer, settlement, and stability of embankments founded on columns installed by deep mixing methods," National Technical University of Athens School of Civil Engineering Geotechnical Department - Foundation Engineering Laboratory, 2007.

[8] N. N. S. Yapage, D. S. Liyanapathirana, H. G. Poulos, R. B. Kelly, and C. J. Leo, "An investigation of progressive failure of geosynthetic reinforced deep cement mixed column supported embankments," in Proc. Int. conf. on Ground Improvement and Ground Control, Wollongong, Australia, 2012, vol. 2, pp. 1345-1351.

[9] CDIT (Coastal Development Institute of Technology), The Deep Mixing Method: Principle, Design and Construction, A.A. Balkema: The Netherlands, 2002.

[10] Public Work Research Center: Design and Construction Manual on Deep Mixing Method for Inland Construction, 2004, p.326.

[11] D. Karastanev, M. Kitazume, S. Miyajima, and T. Ikeda, "Bearing capacity of shallow foundation on column type DMM improved ground," in Proc. the 14th International Conference on Soil Mechanics and Foundation Engineering, 1997, vol. 3, pp.1621-1624.

[12] H. Hashizume, Y. Okochi, J. Dong, N. Horii, Y. Toyosawa, and S. Tamate, "Study on the behavior of the ground inmrpved using deep mixing method," in Proc. International Conference on Centrifuge 98 , 1998, pp. 851-856.

[13] M. Kitazume, T. Ikeda, S. Miyajima, and D. Karastanev, "Bearing capacity of improved ground with column type DMM," in Proc. the 2nd International Conference on Ground Improvement Geosystems, 1996, vol. 1, pp. 503-508.

[14] M. Kitazume and K. Maruyama, "Internal stability of group column type deep mixing improved ground under embankment loading," Soils and Foundations, vol. 47, no. 3, pp. 323-340, 2007.

[15] M. Kivelo, "Stabilization of embankments on soft soil with lime/cement columns," Ph.D dissertation, Royal Institute of Technology, Sweden, 1998.

[16] B. B Broms, Lime and Lime/Cement Columns: Ground Improvement, 2nd edition, M.P. Moseley and K.Kirsch, Eds. Spon Press, 2004, pp. 252-330.

[17] M. Kitazume, "Stability of group column type DM improved ground under embankment loading behavior of sheet pile quay wall," The port and airport research institute, Nagase, Yokosuka, Japan, vol. 47, no. 1, March 2008.

[18] N. N. S. Yapage, D. S. Liyanapathirana, H. G. Poulos, R. B. Kelly, and C. J. Leo, "An investigation of failure modes for geosynthetic reinforced column supported embankments," in Proc. 65th Canadian Geotechnical Conference, Winnipeg, Manitoba, 2012.

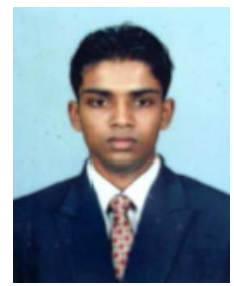

N. N. S. Yapage received his BSc (Hons I) in Civil Engineering from University of Moratuwa, Sri Lanka in 2009. He is currently pursuing his $\mathrm{PhD}$ degree in Geotechnical Engineering in University of Western Sydney, Australia. 


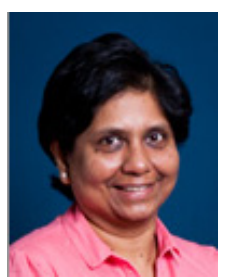

D. S. Liyanapthirana is a senior lecturer in University of Western Sydney, Australia. She completed her $\mathrm{PhD}$ in Geotechnical Engineering at the University of Western Australia in 1999.

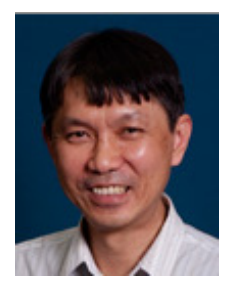

C. J. Leo is an associate professor at the University of Western Sydney. He is a civil engineer with professional engineering experience in infrastructure and geotechnical engineering, including the maintenance, design and construction of roads, road tunnels and slopes.

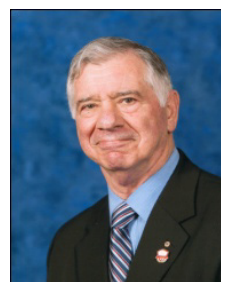

Harry Poulos is a senior principal with Coffey Geotechnics and an Emeritus Professor at the University of Sydney. His main interests are in foundations for tall buildings.

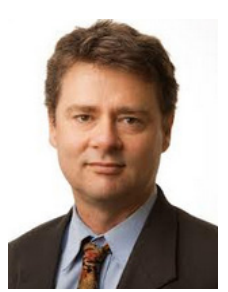

Richard Kelly is a principal geotechnical engineer with Coffey Geotechnics. He completed his $\mathrm{PhD}$ in University of Sydney in 2001. His main research interests are ground improvement and connecting academia to industry: from laboratory to prototype to production. 